\title{
Numerical Investigation of the Photonic-Crystal Fibres with Suspended Core
}

\author{
T. Pustelny* And M. Grabka \\ Department of Optoelectronics at Institute of Physics, Silesian University of Technology \\ Krzywoustego 2, 44-100 Gliwice, Poland
}

\begin{abstract}
The photonic-crystal fibre with a suspended core was analyzed in order to explain possibilities of its using in investigation of electrooptic and magnetooptic effects occurring in the molecules of gas passed into air holes in the fibre. As results from numerical analysis, even $20 \%$ of electromagnetic optical energy can be propagating in the area of the air holes. The obtained results indicate the possibility of scanning the electrooptic and magnetooptic effects in photonic-crystal fibres with a suspended core in the case of considerable diminishing of the core (even below $1 \mu \mathrm{m}$ ) or in the case of light applying with its larger wavelengths.
\end{abstract}

PACS numbers: 42.70.Qs, 42.81.Qb, 42.81.-i, 42.81.Pa, 07.60.Vg

\section{Introduction}

Index-guided photonic-crystal fibres operate basing on the modified principle of total internal reflection (MTIR) [1]. The complex structure of the photonic-crystal fibre may be approximately treated as ranges of some definite averaged value of the refractive index. The structure of this kind can be described basing on the effect of total internal reflection. In many cases such an approximation permits to explain the behaviour of the electromagnetic field in photonic-crystal fibres. In Refs. [2-8] quite a number of phenomena, characterizing this kind of photonic-crystal fibres, have been observed and described. Particularly interesting is the effect of increasing of attenuation of optical waves when their wavelengths are being reduced [5-8].

Of very importance is here the fact that in the mechanism of leading the light can take into consideration the averaged value of the refractive index of the photonic structure in photonic-crystal fibres. Therefore, structures composed of only one material can be obtained, in which air holes are provided $[2,8]$. The core, however, must still form the area with an increased value of refractive index. Many configurations of these fibres have been obtained, particularly fibres with a suspended core, consisting of small core surrounded by several very large air holes, whose dimensions are some tens $\mu$ m order [2].

In the case of another class of photonic-crystal waveguides - fibres with a photonic forbidden band gap (PBGF), the light can be propagating in the air core. Concerning PBGF fibres of the honeycomb type $[2,8]$ attempts were undertaken to introduce into the core gas

* corresponding author; e-mail: tpustelny@polsl.pl molecules, which permitted to investigate Raman's scattering effect by means of fibers [5-7].

As has been proved in [2], in the case of PBGF fibres up to $98.8 \%$ of the optical energy can be propagating in the air core.

The question arises whether in photonic-crystal fibres with a suspended core electrooptical and magnetooptical phenomena can be investigated [9-15]. This would require the interaction of the optical energy, leaking out from the core, with molecules of the gaseous medium supplied to the air holes. One of these possibilities is to modify the structure of the fibre in such a way that the electromagnetic energy of optical wave would be pushed out of the glass core - e.g. by modification the core such that its refractive index would have smaller value. But as has been proved, even in the cases of essential changes of the refractive index of the core, leakage of the optical energy rating into the air holes is rather limited $[3,8]$. The present paper deals with a numerical analysis of the amount of optical energy propagating in the glass core and in the air holes of photonic-crystal fibres with suspended core.

\section{Numerical analysis}

The analyzed fibre is schematically shown in Fig. 1. It is a typical photonic fibre with a suspended core. The fibres of this kind were fabricated in the Laboratory of Optical Fibres Technology at the University of Lublin, Poland. Just such fibres were analysed numerically below.

Dimensions of the analyzed fibres:

- total diameter of the fibre: $130 \mu \mathrm{m}$,

- size of the air holes: ca. $50 \mu \mathrm{m}$ by $35 \mu \mathrm{m}$, 


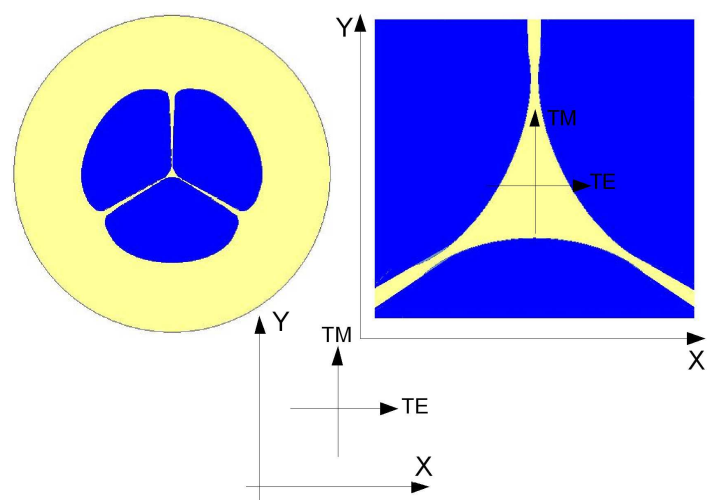

Fig. 1. Cross-section of the analysed fibre (left); enlarged image of the core (right).

- width of the glass bridges (between the air holes): $0.5-1.0 \mu \mathrm{m}$,

- radius $r$ (dimensions) of the suspended core: ca. $2.25 \mu \mathrm{m}$,

- length of the fibre: $1 \mathrm{~m}$.

As Maxwell's equations are independent of the scale, i.e. of the ratio $\lambda / r(\lambda$ - wavelength, $r$ - characteristic dimension of the structure, e.g. - dimension of the core), the wavelength can be normalized, providing more interesting information about wider class of analyzed photonic structures. The refractive index of the core (the glass) was assumed to have a constant value $n=1.44$. The analysis does not take into consideration material dispersion nor material attenuation. Keeping in mind the very small amount of optical power propagating in modes of higher orders, the analysis concerns merely polarized modes of the first order (excited by a Gaussian beam). The following denotations have been accepted (Fig. 1): TM for the fundamental mode with vertical polarisation $(Y)$, and TE for the mode with horizontal polarisation $(X)$.

\section{Results}

Numerical analysis of light propagation into the fibres was performed using the Optical Design Software System of OPTIWAVE SYSTEM Incorporation (Canada).

Thanks to the application of numerical analysis it has become possible to get the relation of the effective refractive index of the mode as a function of the normalized wavelength $\lambda / r$ (Fig. 2).

For the value of core $r=2.25 \mu \mathrm{m}$ additionally the dependence of the effective modal index in the function on the wavelength has been presented (Fig. 3). The effective refractive index of the mode drops, due to the increasing leakage of the modal electromagnetic field into the zone of air holes. Thus the refractive index of the air holes is gaining more and more influence on the effective refractive index of the structure.

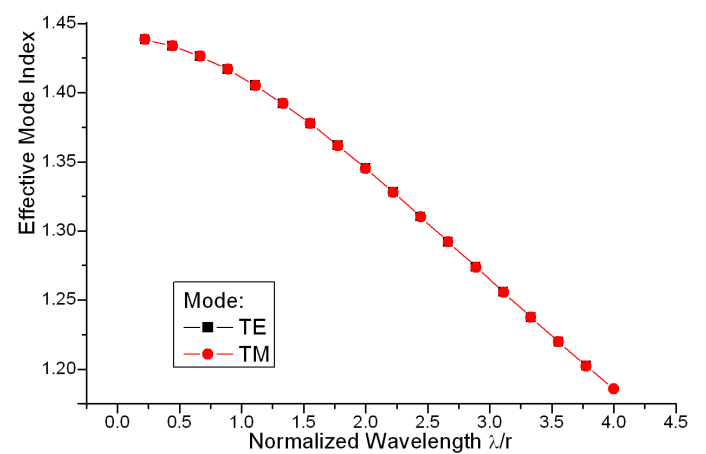

Fig. 2. Effective refractive index of the modes TE and TM.

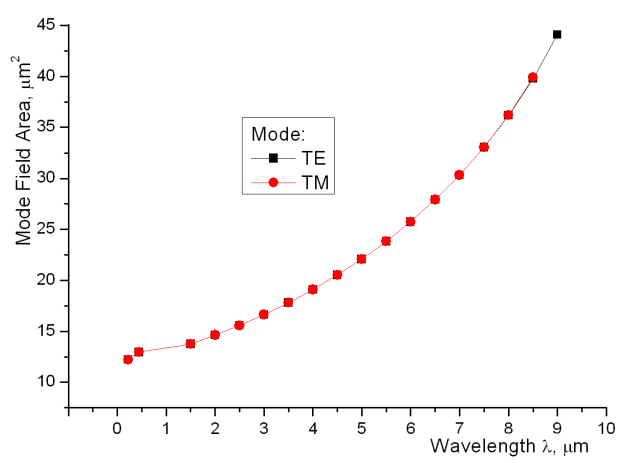

Fig. 3. Size of the modal field in the wavelength function at $r=2.25 \mu \mathrm{m}$.

Generally both modes display identical relations. Discrepancies can be observed in the case of larger wavelengths.

Figure 4 illustrates the modal field at the ratio $\lambda / r=3.3$. It can be seen that the mode TM leaks stronger into the two upper air holes, whereas the mode TE leaks mainly into the air hole at the bottom of the structure. This may become important when the gas is passed into one of the air holes and the fibre is excited by polarized light. The numerical analysis has shown that the total optical energy passing through the area of air holes (Fig. 5) is very similar in both modes TE and TM. As one can see, when the wavelength $\lambda$ equals the diameter of the core (e.g. $\lambda=2.25 \mu \mathrm{m}$ and $r=1.125 \mu \mathrm{m}$ ), the optical energy passing through the air holes amounts to about $5 \%$. For $\lambda / r=3$ in the air holes there propagates near $15 \%$ of electromagnetic energy, which may form conditions for observing the magnetooptic effects in selected gases located in the holes of such photonic-crystal fibre.

A separate and important problem is the possibility of coupling a sufficient amount of optical energy to fundamental mode. Figure 3 indicates that the modal field is of order of several score of $\mu \mathrm{m}^{2}$. Figure 6 provides the exemplary characteristic (for $\lambda / r=1 / 3$ ) of coupling the power between the fundamental mode TM and the diameter of the incident Gaussian beam. The maximum of coupling occurs, of course, in the case of an accurate 


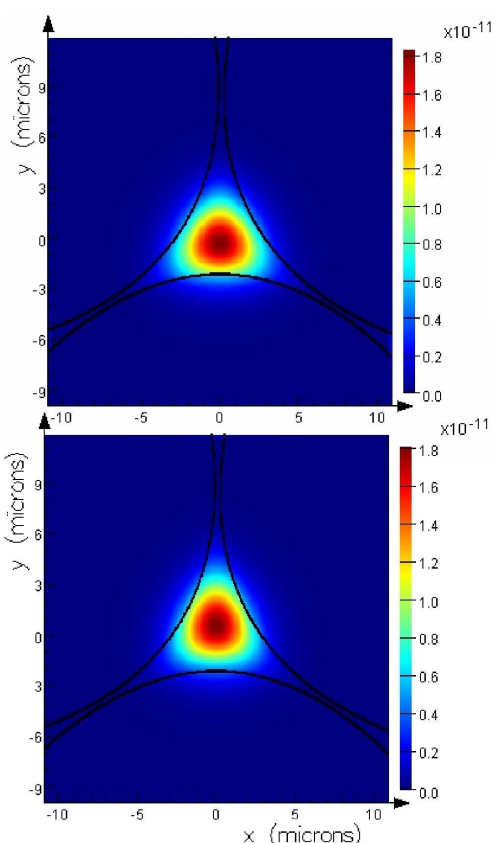

Fig. 4. Modal field concerning the modes TE (top) and TM (bottom), the obtained results concern $\lambda / r=3.3$.

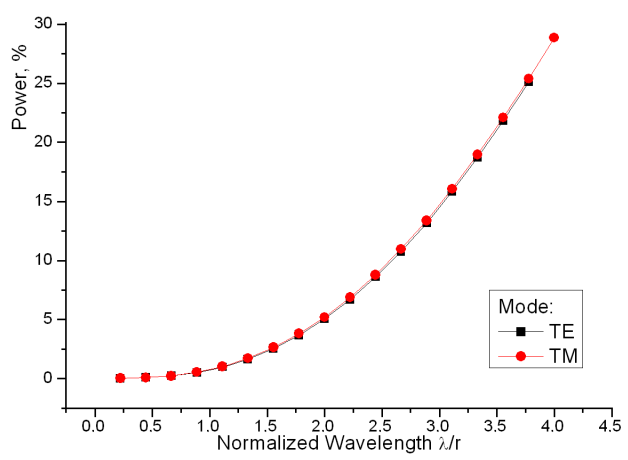

Fig. 5. Amount of optical energy passing through the area of air holes.

matching of the size of the modal area with the area of the Gaussian beam. The growing part of the diagram arises from lacking of modal matching of the source of light (the Gaussian beam) and the fibre, whereas the decreasing part is due to the too large modal area of the light beam, so that some part of the optical energy does not introduce into the core. It is essentially important that at the most $95 \%$ of the optical energy can be introduced into the fundamental mode. The remaining part of the energy is reflected on the front of fibre and gets to modes of higher orders, localized to a considerably higher rate in the area of the air holes. (As the higher order modes are only weakly excited, they have not been analyzed in the presented paper.) In the case of exciting the whole fibre with the total diameter of $130 \mu \mathrm{m}$ (the area of the modal field of the incidence beam being equal to the total area of the cross-section of the fibre) $0.1 \div 0.01 \%$ of optical energy is coupled to the fundamental mode. Such analyses have also been performed concerning other values of normalized wavelengths $\lambda / r$. The very interesting results have been gathered in Fig. $7 \mathrm{a}$ and $\mathrm{b}$.

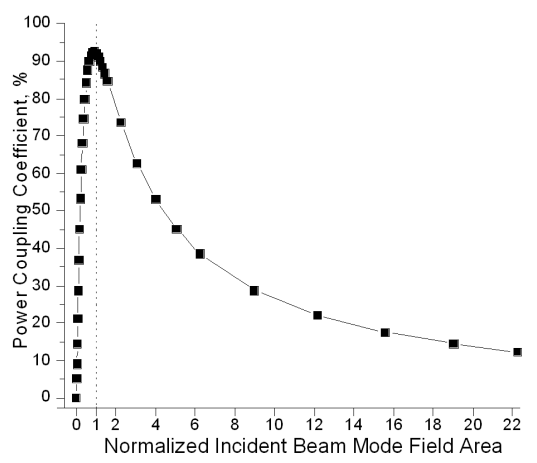

Fig. 6. Coefficient of coupling of the Gauss beam directed towards the fundamental mode TM; $\lambda / r=1 / 3$.

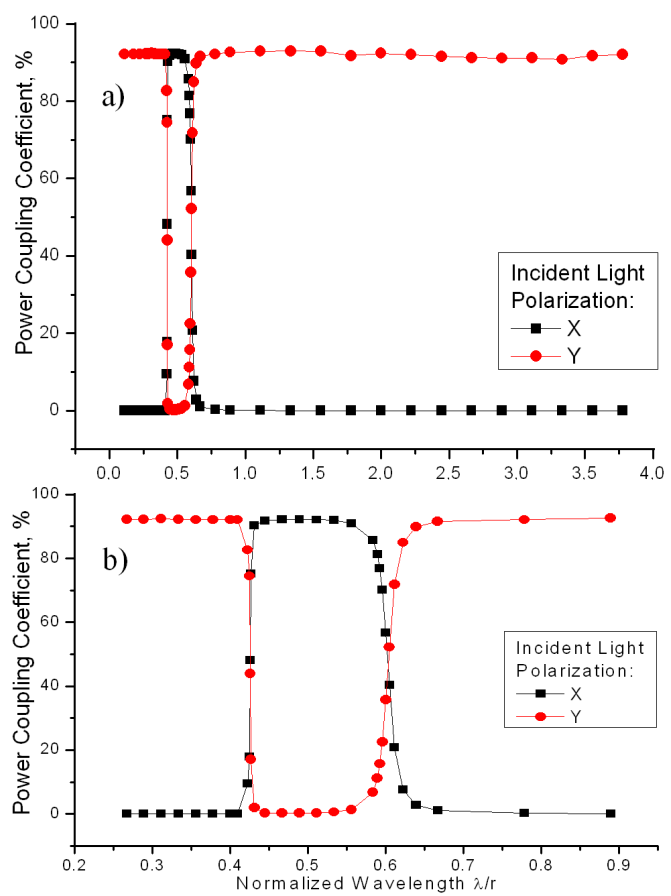

Fig. 7. (a) Coupling coefficient of the power depending on the normalized wavelength $\lambda / r$. (b) The range of changes of the polarization of the mode TM is enlarged.

The level of coupling attains the value of 95\%, independently of the normalized wavelengths. It ought to be mentioned that in the analyzed photonic-crystal fibre for some values of $\lambda / r(\lambda / r \approx 0.4 \div 06)$ changes in the polarization of the mode TM into the mode TE were to be observed (Fig. 7a and b).

The numerical investigation has shown that the $\lambda / r$ range in which the polarization of propagating light changes with TM in TE depends on the value of refrac- 
tive index of the suspended core in the photonic-crystal fibre.

\section{Conclusions}

The photonic-crystal fibre with a suspended core was analyzed in order to explain possibilities of its using in investigation of electrooptic and magnetooptic effects occurring in the molecules of gas passed into air holes in the fibre. As results from numerical analysis, even $20 \%$ of electromagnetic optical energy can be propagating in the area of the air holes. A change in polarization from the TM to TE modes in some $\lambda / r$ range was observed, which is of importance in the case of exciting the fibre by means of polarized light.

The obtained results indicate the possibility of scanning the electrooptic and magnetooptic effects in photonic-crystal fibres with a suspended core in the case of considerable diminishing of the core (even below $1 \mu \mathrm{m}$ ) or in the case of light applying with larger wavelengths.

The experimental results of investigation of such kind of photonic-crystal fibre will be published.

\section{Acknowledgments}

The work was sponsored by the State Committee for Scientific Research within the grant No. N510 387735.

\section{References}

[1] T. Pustelny, Physical and Technical Aspects of Optoelectronic Sensors, Ed. SUT, Gliwice 2005, p. 86.

[2] P. Russel, Science 299, 358 (2003).

[3] T.P. Hansen, J. Broeng, S.E.B. Libori, E. Knudsen, A. Bjarklev, J.R. Jensen, H. Simonsen, IEEE Photon. Technol. Lett. 13, 588 (2001).
[4] L. Xiao, W. Jin, M.S. Demojan, Opt. Express 15, 15637 (2007)

[5] E. Arimondo, in: Progress in Optics, Vol. XXXV, Ed. E. Wolf, Elsevier Science B.V., New York 1996, p. 259.

[6] D.F. Phillips, A. Fleischhauer, A. Mair, R.L. Walsworth, Phys. Rev. Lett. 86, 783 (2001).

[7] C. Liu, Nature 409, 490 (2001).

[8] T. Pustelny, M. Grabka, Acta Phys. Pol. A 114, A-113 (2008).

[9] W. Gawlik, S. Pustelny, in: New Trends in Quantum Coherence and Nonlinear Optics, Ed. R. Drampyan, Nova Sci. Publ., New York 2009, p. 57.

[10] D. Budker, D.F. Kimball, S.M. Rochester, V.V. Yashchuk, M. Zolotorev, Phys. Rev. A 62, 043403 (2000).

[11] S. Pustelny, A. Wojciechowski, M. Gring, M. Kotyrba, J. Zachorowski, W. Gawlik, J. Appl. Phys. 103 063108 (2008).

[12] W. Gawlik, L. Krzemien, S. Pustelny, D. Sangla, J. Zachorowski, M. Graf, A.O. Sushkov, D. Budker, Appl. Phys. Lett. 88, 131108 (2006).

[13] K. Barczak, T. Pustelny, D. Dorosz, J. Dorosz, Europ. Phys. J.-Spec. Top. 154, 11 (2008).

[14] M.P. Ledbetter, V.M. Acosta, S.M. Rochester, D. Budker, S. Pustelny, Phys. Rev. A 75, 023405 (2007).

[15] D. Kasprzak, M. Błahut, E. Maciak, Eur. Phys. J. 154, 113 (2008). 